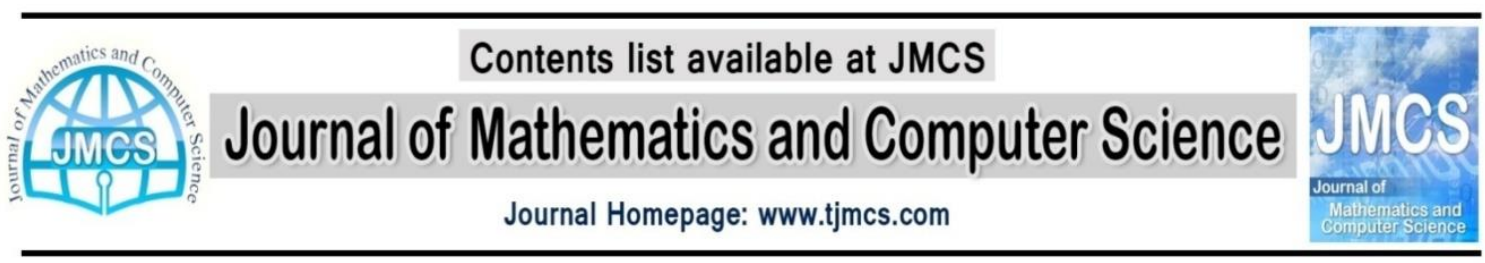

\title{
Levenberg-Marquardt Method for Solving the Inverse Heat Transfer Problems
}

\author{
Nasibeh Asa Golsorkhi, Hojat Ahsani Tehrani \\ Department of mathematics, Shahrood University, Shahrood, Iran. \\ nasibeh_asa@yahoo.com \\ hahsani@shahroodut.ac.ir
}

Article history:

Received August 2014

Accepted October 2014

Available online October 2014

\begin{abstract}
In this paper, The Levenberg-Marquardt method is used in order to solve the inverse heat conduction problem. One-dimensional formulation of heat conduction problem was used. The direct problem was solved with finite-volumes by using an implicit discretization in time. Simulated measurements are obtained from the solution of the direct Problem at the sensor location. Results obtained in this inverse problem will be justified based on the numerical experiments. The results show that the speed of convergence is considerably fast and The Levenberg-Marquardt method is an accurate and stable method to determine the strength of the heat source in the inverse heat conduction problems.
\end{abstract}

Keywords: Levenberg-Marquardt method, inverse problem, heat conduction.

\section{Introduction}

Levenberg-Marquardt Method for Parameter Estimation is an iterative method for solving nonlinear least squaresproblems of parameter estimation. The technique was first derived by Levenberg [1] in 1944, by modifying the ordinary least squares norm. Later, in 1963, Marquardt [2] derived basically the same technique by using a different approach. Marquardt's intention was to obtain a method that would tend to the Gauss method in the neighborhood of the minimum of the ordinary least squares norm, and would tend to the steepest descent method in the neighborhood of the initial guess used for the iterative procedure [1-4]. The so called Levenberg-Marquardt Method [1-6] has been applied to the 
solution of a variety of inverse problems involving the estimation of unknown parameters. The solution of inveres parameter estimation problems by Levenberg-Marquardt Method requires the computation of sensitivity matrix [7]. This Technique is quite efficient for solving linear and nonlinear parameter estimation problems. However, difficulties may arise in nonlinear estimation problems involving a large number of unknown parameters, because of the time spent in the computation of the sensitivity matrix.

The Levenberg-Marquardt method described in [8] is an efficient optimization method for inverse problems of different sorts. The inverse heat conduction problem (IHCP) is concerned with the determination of the thermophysical properties of the medium, the initial or/and boundary temperature distribution for the given temperature field of the unsteady heat conduction $[9,10]$. inverse problem method uses the knowledge of the transient temperature field of the solid

in conjunction with the heat conduction equation and Levenberg-Marquardt algorithm to determine the thermophysical properties more accurately [7]. Kuye and et al. determined the thermal conductivity and the specific heat capacity of neem seeds by least square method in conjunction with Levenberg-Marquardt algorithm [11]. Jose Pujo presented The solution of nonlinear inverse problems and the Levenberg-Marquardt method which will help the reader gain a better understanding of what happens when solving nonlinear problems [12]. Bondarenko and Ivaschenko define inverse problems as the restoration of the medium key parameters including the anomalous diffusion index that correlates with the fractional dimension of the medium and present the numerical solutions obtained by means of the Levenberg-Marquardt method [13].

In this paper, a general method is proposed to determine the strength of the heat source in heat conduction problems. The present inverse problem is solved by using the LevenbergMarquardt method of minimization of the least-Squares norm. The direct problem was solved with finite-volumesb [14,15] by using an implicit discretization in time. Simulated measurements are obtained from the solution of the direct Problem at the sensor location. We present the numerical solutions obtained by means of the Levenberg-Marquardt method and discuss the corresponding result.

\section{The Direct Problem}

Consider the linear transient heat conduction in a plate of unitary dimensionless thickness. The plate is initially at zero temperature and both boundaries at $x=0$ and $x=L$ are kept insulated. For times $t>0$, a plane heat source of strength $g_{p}(t)$ per unit area, placed in the mid-plane $x=0.5$, releases its energy.

The mathematical formulation of this heat conduction problem is given in dimensionless form by:

$$
\begin{array}{lc}
\frac{\partial^{2} T(x, t)}{\partial x^{2}}+g_{p}(t) \delta(x-0.5)=\frac{\partial T(x, t)}{\partial t} & 0<x<1 \text { for } t>0 \\
\frac{\partial T(0, t)}{\partial x}=0 & x=0 \text { for } t>0
\end{array}
$$




$$
\begin{aligned}
& \frac{\partial T(1, t)}{\partial x}=0 \\
& T(x, 0)=0
\end{aligned}
$$

$$
\begin{gathered}
x=1 \text { for } t>0 \\
t=0 \text { in } 0<x<1
\end{gathered}
$$

Where $\boldsymbol{\delta}($.$) is the Dirac delta function.In the Direct Problem associated with the physical$ problem described above, the time-varying strength $g_{p}(t)$ of the plane heat source is known. The objective of the direct problem is then to determine the transient temperature field $T(x, t)$ in the plate.

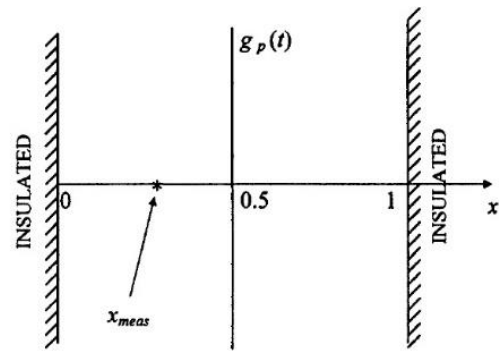

Figure1: Geometry and coordinates for a plane heat source $g_{p}(t)$.

\section{The Inverse Problem}

For the Inverse Problem considered of interest here, the time-varying strength $g_{p}(t)$ of the plane heat source is regarded as unknown. The additional information obtained from transient temperature measurements taken at alocation $x=x_{\text {meas }}$ at times $t_{i}, i=1,2, \ldots, I$, is then used for the estimation of $g_{p}(t)$.

For the solution of the present inverse problem, we consider the unknown energy generation function $g_{p}(t)$ to be parameterized in the following general linear form:

$$
g_{p}(t)=\sum_{j=1}^{N} P_{j} C_{j}(t)
$$

Here, $P$, are unknown parameters and $C_{j}(t)$ are known trial functions (e.g., polynomials, BSplines, etc). In addition, the total number of parameters, $N$, is specified. The problem given by equations (1) with $g_{p}(t)$ unknown, but parameterized as given by equation (2), is an inverse heat conduction problem in which the coefficients $P$, are to be estimated.

The solution of this inverse heat conduction problem for the estimation of the $N$ unknown parameters $p_{j}, j=1, \ldots, N$ is based on the minimization of the ordinary least squares norm given by:

$$
S_{P}=\sum_{i=1}^{I}\left[Y_{i}-T_{i}(P)\right]^{2}
$$

where $S$ is the sum of squares error or objective function, $p^{T}=\left[p_{1}, p_{2}, . . p_{I}\right]$ is the vector of unknown parameters, $T_{i}(P)=T\left(P, t_{i}\right)$ and $Y_{i}=Y\left(t_{i}\right)$ are the estimated temperature and measured temperatureat at time $t_{i}$. The estimated temperatures $T_{i}(P)$ are obtained from the 
solution of the direct problem at the measurement location, $x_{\text {meas }}$ by using the current estimate for the unknown parameters $P_{j}, j=1, \ldots, N$.

\section{The Iterative Procedure}

To minimize the least squares norm given by equations (3), we need to equate to zero the derivatives of $S(P)$ with respect to each of the unknown parameters $\left[p_{1}, p_{2}, . . p_{I}\right]$, Such necessary condition for the minimization of $S(P)$ can be represented in matrix notation by equating the gradient of $S(P)$ with respect to the vector of parameters $P$ to zero, that is,

Where

$$
\nabla S(P)=2\left[-\frac{\partial T^{T}(P)}{\partial P}\right][Y-T(P)]=0
$$

$$
\frac{\partial T^{T}(P)}{\partial P}=\left[\begin{array}{c}
\frac{\partial}{\partial P_{1}} \\
\frac{\partial}{\partial P_{2}} \\
\vdots \\
\frac{\partial}{\partial P_{N}}
\end{array}\right]\left[T_{1}, T_{2}, \ldots, T_{N}\right]
$$

The Sensitivity or Jacobian matrix, $J(P)$, is defined as the transpose of equation (5), that the sensitivity matrix is written as:

$$
J(P)=\left[\frac{\partial T^{T}(P)}{\partial P}\right]^{T}=\left[\begin{array}{ccccc}
\frac{\partial T_{1}}{\partial P_{1}} \frac{\partial T_{1}}{\partial P_{2}} \frac{\partial T_{1}}{\partial P_{3}} & \cdots & \frac{\partial T_{1}}{\partial P_{N}} \\
\frac{\partial T_{2}}{\partial P_{1}} \frac{\partial T_{2}}{\partial P_{2}} \frac{\partial T_{2}}{\partial P_{3}} & \cdots & \frac{\partial T_{2}}{\partial P_{N}} \\
\vdots & \vdots & \vdots & \ldots & \vdots \\
\frac{\partial T_{I}}{\partial P_{1}} \frac{\partial T_{I}}{\partial P_{2}} \frac{\partial T_{I}}{\partial P_{3}} & \cdots & \frac{\partial T_{I}}{\partial P_{N}}
\end{array}\right]
$$

Where $N$ is total number of unknown parameters and $I$ is total number of measurements. The elements of the sensitivity matrix are called the Sensitiviry Coefficients. The sensitivity coefficient $J$, is thus defined as the first derivative of the estimated temperature at time $t$, with respect to the unknown parameter $P_{j}$, that is,

$$
J_{i j}=\frac{\partial T_{i}}{\partial P_{j}}
$$

By using the definition of the sensitivity matrix given by equation (6), equation (4) becomes:

$$
-2 J^{T}(P)[Y-T(P)]=0
$$

The solution of equation (8) for nonlinear estimation problems then requires an iterative procedure, which is obtained by linearizing the vector of estimated temperatures, $T(P)$, with a 
Taylor series expansion around the current solution at iteration k. Such a linearization is given by:

$$
T(P)=T\left(P^{k}\right)+J^{k}\left(P-P^{k}\right)
$$

where $T\left(p^{k}\right)$ and $J^{k}$ are the estimated temperatures and the sensitivity matrix evaluated at iteration $k$, respectively. Equation (9) is substituted into equation (8) and the resulting expression is rearranged to yield the following iterative procedure to obtain the vector of unknown parameters $P[4]$ :

$$
P^{K+1}=P^{K}+\left[\left(J^{K}\right)^{T} J^{K} J^{-1}\left(J^{k}\right)^{T}\left[Y-T\left(P^{K}\right)\right]\right]
$$

Such method is actually an approximation for the Newton (or Newton-Raphson) method [3]. We note that equation (10), require the matrix $J^{T} J$ to be nonsingular, or

$$
\left|J^{\mathrm{T}} \mathrm{J}\right| \neq 0
$$

where $|$.$| is the determinant.$

Equation (11) gives the so called identifiability Condition, that is, if the determinant of $J^{T} J$ is zero, or even very small, the parameters $P_{j}$, for $j=1, \ldots, N$, cannot be determined by using the iterative procedure of equation (10). Problems satisfying $\left|J^{T} J\right|=0$ are denoted illconditioned. Inverse heat transfer problems are generally very ill-conditioned, especially near the initial guess used for the unknown parameters, creating difficulties in the application of equation (10). The Levenberg-Marquardt Method [7] alleviates such difficulties by utilizing an iterative procedure in the form:

$$
\left.P^{K+1}=P^{K}+\left[\left(J^{K}\right)^{T} J^{K}+\mu^{K} \Omega^{k}\right]^{-1}\left(J^{k}\right)^{T}\left[Y-T\left(P^{K}\right)\right]\right]
$$

Where $\mu^{k}$ is a positive scalar named dampingparameter, and $\Omega^{k}$ is a diagonal matrix. The purposeof the matrix term $\mu^{k} \Omega^{k}$, included in equation (12), is to damp oscillations and instabilities due to the ill-conditioned character of the problem, by making its components large as compared to those of $J^{T} J$ if necessary. The damping parameter is made larg in the beginning of the iterations, since the problem is generally ill-conditioned in the region around the initial guess used for the iterative procedure, which can be quite far from the exact parameters. With such an approach the matrix $J^{T} J$ is not required to be non-singular in the beginning of iterations and the Levenberg-Marquardt method tends to the Steepest Descent method, that is, a very small step is taken in the negative gradient direction. The parameter $\mu^{k}$ is then gradually reduced as the iteration procedure advances to the solution of the parameter estimation problem, and then the Levenberg-Marquardt method tends to the Gauss method given by equation (10) [4].

The following criterion were suggested by Dennis and Schnabel [5] to stop the iterative procedure of the Levenberg-Marquardt Method given by equation (12):

$$
\left\|P^{K+1}-P^{K}\right\|<\varepsilon
$$

Where $\varepsilon$ is user prescribed tolerances and $\|$.$\| is the vector Euclidean norm, i.e., \|X\|=$ $\left(X^{T} X\right)^{1 / 2}$, where the superscript $T$ denotes transpose.

\section{Computational Algorithm}


Different versions of the Levenberg-Marquardt method can be found in the literature, depending on the choice of the diagonal matrix $\Omega^{k}$ and on the form chosen for the variation of tte damping parameter $\mu^{k}$ [1-6]. We illustrate here a procedure with the matrix $\Omega^{k}$ taken as:

$$
\Omega^{k}=\operatorname{diag}\left[\left(J^{K}\right)^{T} J^{K}\right]
$$

Suppose that temperature measurements $Y=\left(Y_{1}, Y_{2}, \ldots, Y_{I}\right)$ are given at times $t_{i}, i=1, \ldots, I$. Also, suppose an initial guess $\mathrm{P}^{0}$ is available for the vector of unknown parameters $\mathrm{P}$. Choose a value for $\mu^{0}$, say, $\mu^{0}=0.001$ and set $k=0$ [6]. Then

Step 1. Solve the direct heat transfer problem given by equations (1) with the available estimate $P^{k}$ in order to obtain the temperature vector $T\left(P^{K}\right)=\left(T_{1}, \ldots, T_{I}\right)$.

Step 2. Compute $\mathrm{S}\left(\mathrm{P}^{\mathrm{k}}\right)$ fiom equation (3).

Step 3. Compute the sensitivitymatrix $J^{k}$ defined by equation (6) and thenthe matrix $\Omega^{k}$ given by equation (14), by using the current values of $P^{k}$.

Step 4. Solve the following linear system of algebraic equations, obtained from the iterative procedure of the Levenberg-Marquardt Method, equation (12):

$$
\left[\left(J^{K}\right)^{T} J^{K}+\mu^{K} \Omega^{k}\right] \Delta P^{k}=\left(J^{k}\right)^{T}\left[Y-T\left(P^{K}\right)\right]
$$

in order to compute $\Delta P^{k}=P^{K+1}-P^{K}$.

Step 5. Compute the new estimate $P^{k+1}$ as

$$
P^{K+1}=P^{K+1}-P^{K}
$$

Step 6. Solve the direct problem (1) with the new estimate $P^{k+1}$ in order to find $T\left(P^{k+1}\right)$. Then compute $S\left(P^{k+1}\right)$, as defined by equation (3).

Step 7. 1f $S\left(P^{k+1}\right)>S\left(P^{k}\right)$, replace $\mu^{k}$ by $10 \mu^{k}$ and return to step 4 .

Step 8. If $S\left(P^{k+1}\right)<S\left(P^{k}\right)$,accept the new estimate $P^{k+1}$ and replace $\mu^{k}$ by $0.1 \mu^{k}$.

Step 9. Check the stopping criterion given by equations (13). Stop the iterative procedure if any of them is satisfied. otherwise, replace $k$ by $k+1$ and return to step 3 .

\section{Results and Discussion}

In this section, we present the results obtained with this technique as applied to the solution of our test-problem, involving the estimation of the strength of the heat source. Two different source functions over temporal domain; namely, a sinusoidal function and a triangular function are adopted to illustrate the numerical modeling. The exact temperature and the source strength used in the following examples are selected so that these functions can satisfy equation (1). The accuracy is assessed by the comparison between the estimated and preset source strength. 
The time evolution in all cases is from 0 to $t_{f}=1.4$. The heat sources are applied at $x=$ 0.5 . During the time interval $0<t<1.4$, we consider available for the inverse analysis 100 transient measurements of a single sensor located at $x_{\text {meas }}=1$.

Levenberg-Marquardt method is applied to the estimation of the undetermined heat source. Simulated measurements are obtained from the solution of the Direct Problem at the sensor location. The application of the proposed approach is demonstrated by the following examples in this present. The strength of the heat source is presented as the time-varying function. Detailed descriptions for the examples are shown as follows:

\section{Example 1:}

\section{Example 2:}

$$
\mathrm{g}_{\mathrm{p}}(\mathrm{t})=1+\sin (\pi \mathrm{t})+\cos (\pi \mathrm{t}) \quad \text { when } 0<t<1.4
$$

$$
g_{p}(t)=\left\{\begin{array}{ccc}
0.4+\frac{6}{7} t & \text { when } & 0<t<0.7 \\
1.5-\frac{5}{7} t & \text { when } & 0.7<t<1.4
\end{array}\right.
$$

The solution of the direct problem (1) at the measurement location $x_{\text {meas }}=1$, by using the heat source given by equations (16) and (17), provide the exact (errorless) measurements $Y_{e x}\left(t_{i}\right), i=1, \ldots, I$. Measurements containing random errors are simulated by adding an error term to $Y_{e x}\left(t_{i}\right)$ in the form:

$$
Y\left(t_{i}\right)=Y_{e x}\left(t_{i}\right)+\omega \sigma
$$

where $Y\left(t_{i}\right)$ is simulated measurements containing random errors, $Y_{e x}\left(t_{i}\right)$ is exact (errorless) simulated measurements, $\sigma$ is standard deviation of the measurement errors, $\omega$ is random variable with normal distribution, zero mean and unitary standard deviation. For the $99 \%$ confidence levelwe have $-2.576<\omega<2.576$ [16].

The direct problem Was solved with finite-volumes [14,15] by using an implicit discretization in time. The spatial domain $0<x<1$ was discretized with 100 volumes, while 25 time-steps were used to advance the solutions from $t=0$ to $t_{f}=1.4$. The sensitivity coefficients, were evaluated with finite-differences by utilizing the forward approximation of equation (19) with $\varepsilon=10^{-6}$.

$$
J_{i j} \cong \frac{T_{i}\left(P_{1}, P_{2}, \ldots, P_{j}+\varepsilon P_{j}, \ldots, P_{N}\right)-T_{i}\left(P_{1}, P_{2}, \ldots, P_{j}, \ldots, P_{N}\right)}{\varepsilon P_{j}}
$$

The initial guesses for the unknown functions were taken as zero. Two different levels of measurement errors considered for the analysis included $\sigma=0$ (errorless) and $\sigma=$ $0.01 T_{\max }$ where $T_{\max }$ is the maximum measured temperature. The estimated results without the measurement errors are shown in Figure (2(a)) and (3(a)). All examples have excellent approximations when measurement errors are free. As the random errore $\sigma=0.01 T_{\max }$, the results are shown in figure (2(b)) and (3(b)). This figures reveal that the proposed method is robust and stable when the measurement error is included in the estimation. 


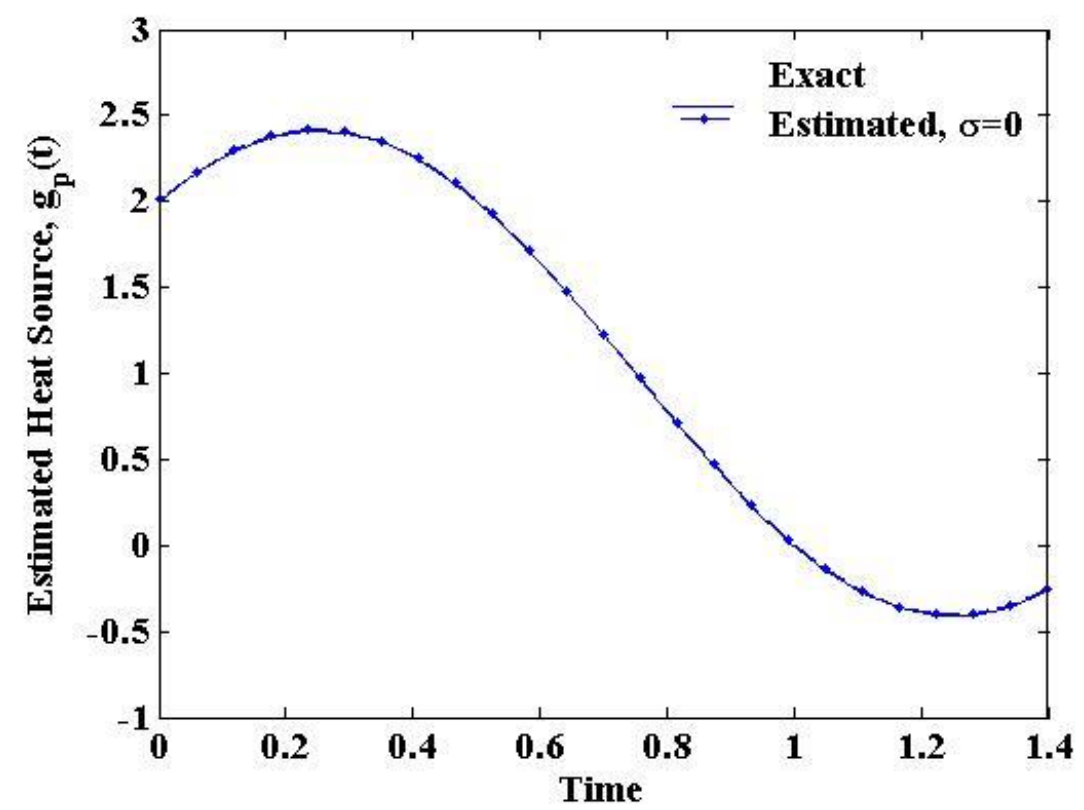

(a) $\sigma=0$

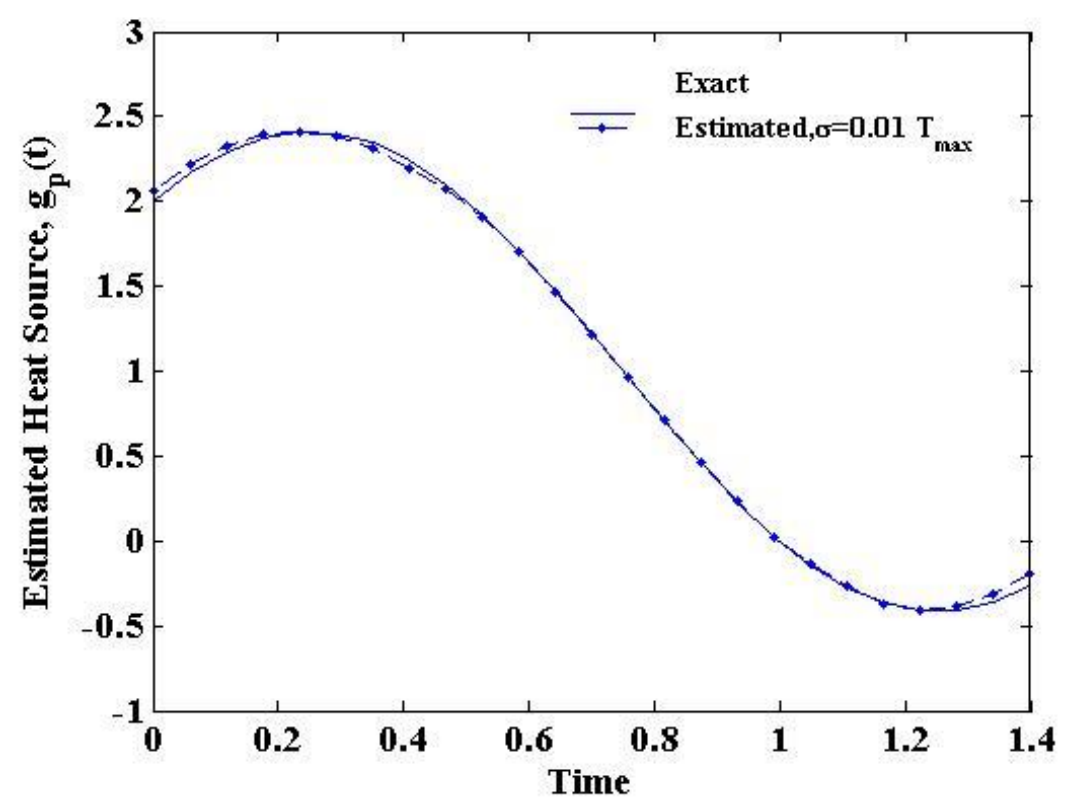

(b) $\sigma=0.01 T_{\max }$

Figure 2: The (a) Estimation of the sinusoidal strength of the heat source with $\sigma=0$ and

(b) Estimation of the sinusoidal strength of the heat source with $\sigma=0.01 T_{\max }$. 


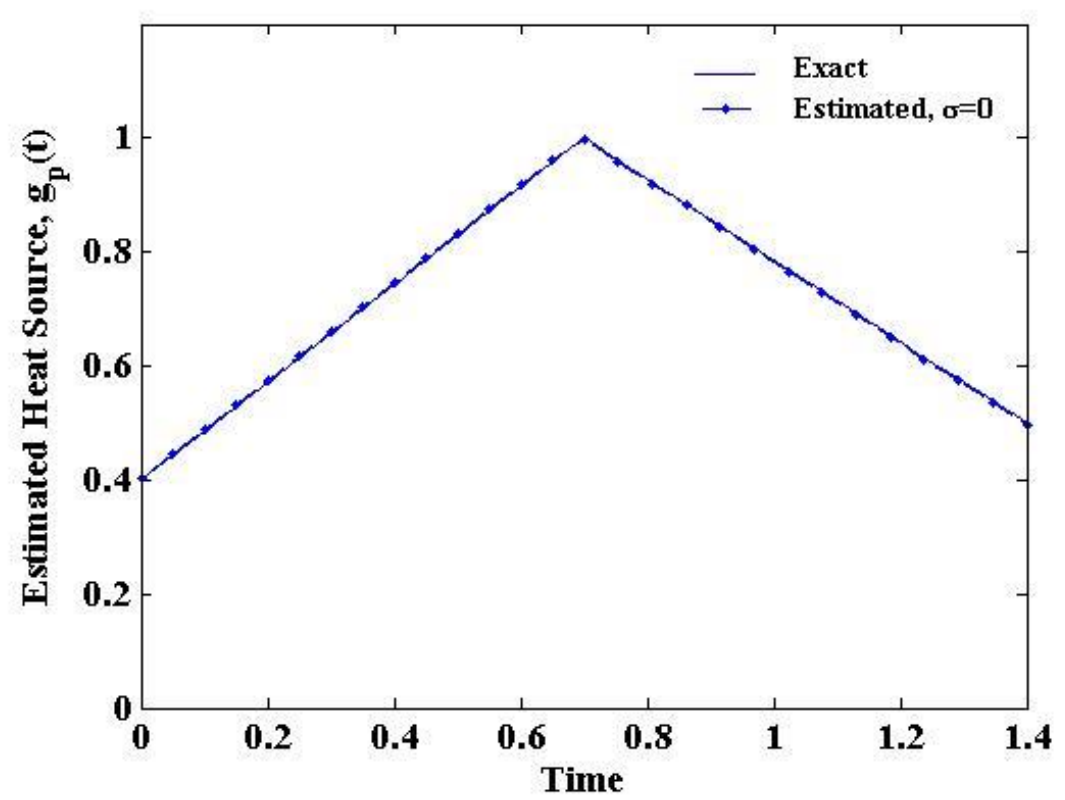

(a) $\sigma=0$

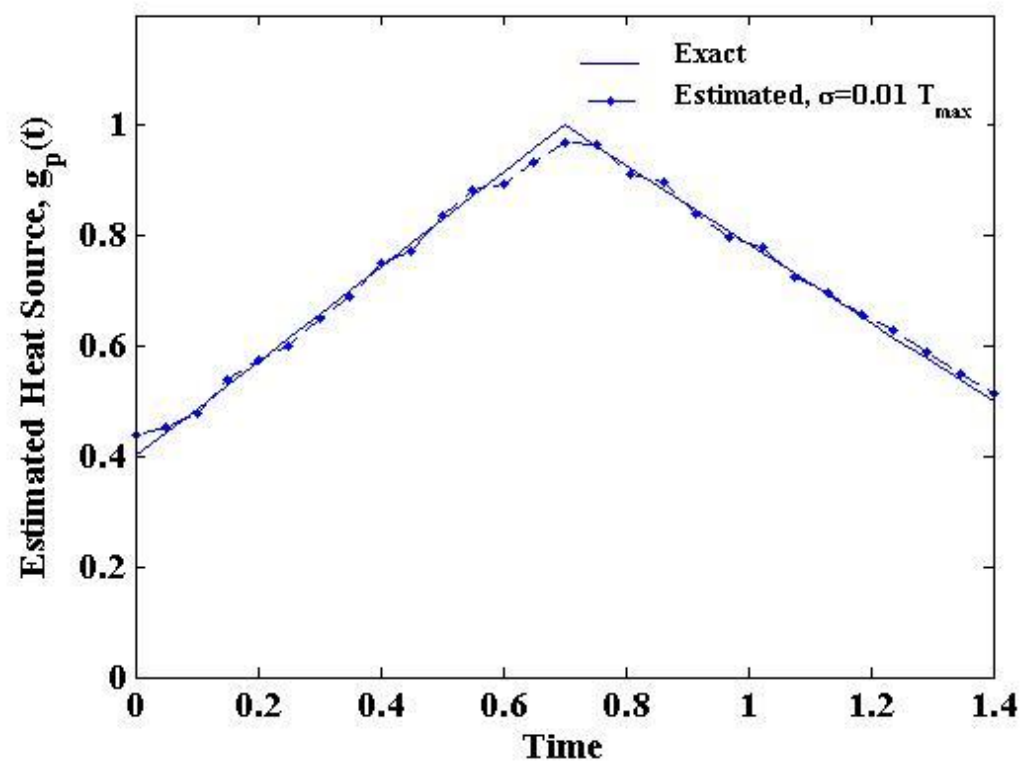

(b) $\sigma=0.01 T_{\max }$

Figure 3: The (a) Estimation of the triangular strength of the heat source with $\sigma=0$ and (b) Estimation of the triangular strength of the heat source with $\sigma=0.01 T_{\max }$

To investigate the deviation of the estimated results from the exact solution, the RMS errors for the estimated solutions are defined as follows:

$$
e_{R M S}=\sqrt{\frac{1}{I} \sum_{i=1}^{I}\left[g_{e s t}\left(t_{i}\right)-g_{e x}\left(t_{i}\right)\right]^{2}}
$$


Where $g_{\text {est }}\left(t_{i}\right)$ is the estimated source term function at time $\mathrm{t}_{\mathrm{i}}, g_{\text {est }}\left(t_{i}\right)$ is the exact source term function (used to generate the simulated measurements) at time $t_{I}$ and $I$ is the number of measurements.

Table1 present the RMS errors and number of iterations for Levenberg-Marquardt technique.

Table 1: Results obtained to approximate the Heat Source function

\begin{tabular}{|c|c|c|c|c|}
\hline \multirow{2}{*}{ Function } & \multicolumn{2}{|c|}{$\mathbf{e}_{\mathrm{RMS}}$} & \multicolumn{2}{c|}{ Number of Itration } \\
\cline { 2 - 5 } & $\boldsymbol{\sigma}=\mathbf{0}$ & $\boldsymbol{\sigma}=\mathbf{0 . 0 1 T _ { \text { max } }}$ & $\boldsymbol{\sigma}=\mathbf{0}$ & $\boldsymbol{\sigma}=\mathbf{0 . 0 1 \boldsymbol { T } _ { \text { max } }}$ \\
\hline Sinusoidal function & 0.0065 & 0.0179 & 4 & 6 \\
\hline triangular function & 0.004 & 0.0201 & 4 & 8 \\
\hline
\end{tabular}

Table1 shows that the exact values are recovered with this technique, when errorless measurements $(\sigma=0)$ were used. In such cases, we had the small number of iterations and for cases involving measurement errors $\left(\sigma=0.01 T_{\max }\right)$, the small RMS error was also obtained. The foregoing analysis reveals that Levenberg-Marquardt technique, provided an accurate estimation. Furthermore, the number of iterations shows that the speed of convergence is fast.

\section{Conclusion}

In this paper we presented the solution of an inverse problem for one-dimentional heat conduction problem. The direct problem was solved with finite-volumes by using an implicit discretization in time. As well, the inverse solution at each time step is solved by LevenbergMarquardt method of minimization of the least-Squares norm. Two examples have been used to show the usage of the proposed method. The results show that the exact solution can be found through the proposed method without measurement errors. The use of simulated temprature measurements containing random error shows that accurate estimates can be obtained for the unknown functions with the present approach. Finally, The results obtained illustrate that the Levenberg-Marquardt method is applicable and efficient for the solution of the inverse heat conduction problem. Moreover, the proposed method is also applicable to the other kinds of inverse problems such as boundary estimation in the one- or multi-dimensional heat transfer problems.

\section{References}

[1] K. Levenberg, A Method for the Solution of Certain Non-linear Problems in Least Squares, Quart. Appl. Math. 2 (1944)164 -168.

[2] D. W. Marquardt, An Algorithm for Least Squares Estimation of Nonlinear Parameters, J. Soc. Ind. Appl. Math. 11 (1963)431-441.

[3] Y. B. Bard, Nonlinear Parameter Estimation, Acad. Press, New York, 1974.

[4] J. V. Beck, and K. J. Arnold, Parameter Estimation in Engineering and Science, Wiley, New York, 1977.

[5] J. Dennis and R. Schnabel, Numerical Methodr for Unconstrained Optimization and Nonlinear Equations, Prentice Hall, 1983. 
N. A. Golsorkhi, H. A. Tehrani / J. Math. Computer Sci. 13 (2014), 300-310

[6] W. H. Press, B. F. Flannery, S. A. Teukolsky and W. T. Wetterling, Numerical Recipes, Cambridge University Press, New York, 1989.

[7] M.N. Ozisik, Heat Conduction, 2nd ed. John Wiley, New York, 1993.

[8] S.R. Arridge and M. Schweiger, A General framework for iterative reconstruction algorithms in optical tomography, using a finite element method, Appl Opt ,42 (1998) 9683-9706.

[9] E. Ghasemi $\square$, A. A. Ranjbar, A. Ramiar , Three-dimensional Numerical Analysis of Heat Transfer Characteristics of Solar Parabolic Collector With Two Segmental Rings, Journal of mathematics and computer Science, 7 (2013) 89 - 100.

[10] H. Heidarzadeh, M. Mashinchi joubari, R. Asghari, Application of Adomian Decomposition Method to Nonlinear Heat Transfer Equation, Journal of mathematics and computer Science, 4.3 (2012) 436 - 447.

[11] A.O. Kuye, C.O.C. Oko and S.N. Nnamchi, Determination of the thermal conductivity and specific heat capacity of neem seeds by inverse problem method, Journal of Engineering Science and Technology Review 3.1 (2010) 1-6.

[12] J. Pujo, The solution of nonlinear inverse problems and the Levenberg-Marquardt method, GEOPHYSICS. 72 (2007) 1-16.

[13] A.N. Bondarenko and D.S. Ivaschenko, Levenberg-Marquardt Method for Restoration of Layered Medium Key Parameters, Russian-Korean International Symposium on Science and Technology (2005) 43- 46.

[14] R. K. LeVeque, Finite Volume Methods for Hyperbolic Problems, Cambridge University Press, 2002.

[15] H. Versteeg and W. Malalasekera, An Introduction to Computational Fluid Dynamics: The Finite Volume Method, Prentice Hall, 2007.

[16] M.N. Ozisik and H. R. B. Orlande, Inverse Heat Transfer Fundamentals and Applications, Taylor and Francis, New York, 2000. 